\title{
THE CHROMATIC NUMBER OF RANDOM INTERSECTION GRAPHS
}

\author{
KATARZYNA RYBARCZYK ${ }^{1}$ \\ Adam Mickiewicz University \\ Faculty of Mathematics and Computer Science \\ 60-769 Poznań, Poland \\ e-mail: kryba@amu.edu.pl
}

\begin{abstract}
We study problems related to the chromatic number of a random intersection graph $\mathcal{G}(n, m, p)$. We introduce two new algorithms which colour $\mathcal{G}(n, m, p)$ with almost optimum number of colours with probability tending to 1 as $n \rightarrow \infty$. Moreover we find a range of parameters for which the chromatic number of $\mathcal{G}(n, m, p)$ asymptotically equals its clique number.
\end{abstract}

Keywords: random intersection graphs, chromatic number, colouring algorithms.

2010 Mathematics Subject Classification: 05C80, 05C15, 05 C85.

\section{REFERENCES}

[1] M. Behrisch, Component evolution in random intersection graphs, Electron. J. Combin. 14 (2007) R17.

[2] M. Behrisch, A. Taraz and M. Ueckerdt, Coloring random intersection graphs and complex networks, SIAM J. Discrete Math. 23 (2009) 288-299. doi:10.1137/050647153

[3] M. Bloznelis, Component evolution in general random intersection graphs, SIAM J. Discrete Math. 24 (2010) 639-654. doi:10.1137/080713756

[4] B. Bollobás, The chromatic number of random graphs, Combinatorica 8 (1988) 49-55. doi:10.1007/BF02122551

\footnotetext{
${ }^{1}$ Katarzyna Rybarczyk acknowledges a support of NCN (Narodowe Centrum Nauki) grant 2014/13/D/ST1/01175.
} 
[5] B. Bollobas and P. Erdős, Cliques in random graphs, Math. Proc. Cambridge Philos. Soc. 80 (1976) 419-427. doi:10.1017/S0305004100053056

[6] J.A. Fill, E.R. Scheinerman and K.B. Singer-Cohen, Random intersection graphs when $m=\omega(n)$ : An equivalence theorem relating the evolution of the $G(n, m, p)$ and $G(n, p)$ models, Random Structures Algorithms 16 (2000) 156-176. doi:10.1002/(SICI)1098-2418(200003)16:2〈156::AID-RSA3)3.0.CO;2-H

[7] A. Frieze and C. McDiarmid, Algorithmic theory of random graphs, Random Structures Algorithms 10 (1997) 5-42. doi:10.1002/(SICI)1098-2418(199701/03)10:1/2 〈5::AID-RSA2 33.0.CO;2-Z

[8] G.R. Grimmett and C.J.H. McDiarmid, On colouring random graphs, Math. Proc. Cambridge Philos. Soc. 77 (1975) 313-324. doi:10.1017/S0305004100051124

[9] S. Janson, T. Łuczak and A. Ruciński, Random Graphs (Wiley, 2001).

[10] M. Karoński, E.R. Scheinerman and K.B. Singer-Cohen, On random intersection graphs: The subgraph problem, Combin. Probab. Comput. 8 (1999) 131-159. doi:10.1017/S0963548398003459

[11] V. Kurauskas and K. Rybarczyk, On the chromatic index of random uniform hypergraphs, SIAM J. Discrete Math. 29 (2015) 541-558. doi:10.1137/130942292

[12] T. Łuczak, The chromatic number of random graphs, Combinatorica 11 (1991) $45-54$. doi:10.1007/BF01375472

[13] S. Nikoletseas, C. Raptopoulos and P.G. Spirakis, Colouring non-sparse random intersection graphs, in: Mathematical Foundations of Computer Science 2009, Lecture Notes in Comput. Sci. 5734, R. Královič, D. Niwiński (Ed(s)), (Springer Berlin Heidelberg, 2009) 600-611. doi:10.1007/978-3-642-03816-7_51

[14] K. Rybarczyk, Equivalence of the random intersection graph and $G(n, p)$, Random Structures Algorithms 38 (2011) 205-234. doi:10.1002/rsa.20356

[15] K. Rybarczyk, Constructions of independent sets in random intersection graphs, Theoret. Comput. Sci. 524 (2014) 103-125. doi:10.1016/j.tcs.2014.01.006

[16] E. Shamir and E. Upfal, Sequential and distributed graph coloring algorithms with performance analysis in random graph spaces, J. Algorithms 5 (1984) 488-501. doi:10.1016/0196-6774(84)90003-8

Received 16 November 2015

Revised 27 September 2016

Accepted 27 September 2016 